Research Article

\title{
Total Face Irregularity Strength of Grid and Wheel Graph under K-Labeling of Type $(1,1,0)$
}

\author{
Aleem Mughal and Noshad Jamil \\ University of Management and Technology, Lahore, Pakistan \\ Correspondence should be addressed to Noshad Jamil; noshad.jamil@umt.edu.pk
}

Received 23 June 2021; Accepted 10 August 2021; Published 31 August 2021

Academic Editor: Muhammad Kamran Siddiqui

Copyright (c) 2021 Aleem Mughal and Noshad Jamil. This is an open access article distributed under the Creative Commons Attribution License, which permits unrestricted use, distribution, and reproduction in any medium, provided the original work is properly cited.

In this study, we used grids and wheel graphs $G=(V, E, F)$, which are simple, finite, plane, and undirected graphs with $V$ as the vertex set, $E$ as the edge set, and $F$ as the face set. The article addresses the problem to find the face irregularity strength of some families of generalized plane graphs under $k$-labeling of type $(\alpha, \beta, \gamma)$. In this labeling, a graph is assigning positive integers to graph vertices, graph edges, or graph faces. A minimum integer $k$ for which a total label of all verteices and edges of a plane graph has distinct face weights is called $k$-labeling of a graph. The integer $k$ is named as total face irregularity strength of the graph and denoted as $\operatorname{tfs}(G)$. We also discussed a special case of total face irregularity strength of plane graphs under $k$-labeling of type $(1,1$, $0)$. The results will be verified by using figures and examples.

\section{Introduction}

This article is based on simple, plane, finite, and undirected graphs $G=(V, E, F)$. Graph labeling is a mapping that maps graph elements $(V, E, F)$ into positive integers, and we name these positive integers as labels. Suppose that $\alpha, \beta, \gamma \in\{0,1\}$ and $k$ is a positive integer, then a branch of labeling, named as, $k$-labeling of type $(\alpha, \beta, \gamma)$, is a mapping $\phi$ from the set of graph elements $(V, E, F)$ into the set of positive integers $\{1,2,3, \ldots, k\}$. A labeling of type $(1,1,0)$ of grid graph $G_{n}^{m}$ means that vertices and edges are labeled but face is not labeled. We will work on labeling of type $(1,1,0)$ for the grid graphs $G_{n}^{m}$, in which the vertices and edges will be labeled but our ultimate focus will be on calculating distinct face weights. A detailed review of graph labeling can be seen in [1].

If the domain of $k$-labeling of type $(\alpha, \beta, \gamma)$ is vertex set, edge set, face set, or vertex-edge set, then we name this as vertex $k$-labeling of type $(1,0,0)$, edge $k$-labeling of type $(0,1,0)$, face $k$-labeling of type $(0,0,1)$, or total $k$-labeling of type $(1,1,0)$, respectively. The other possible cases are vertex-face set, edge-face set, and vertex-edge-face set which we call as vertex-face $k$-labeling of type $(1,0,1)$, edge-face $k$-labeling of type $(0,1,1)$, and entire $k$-labeling of type $(1,1,1)$, respectively. The trivial case $(\alpha, \beta, \gamma)=(0,0,0)$ is not accepted. The weight of any vertex in a graph is the sum of labels of that particular vertex and its adjacent edges. The weight of any edge of a graph is the sum of lables of its adjacent vertices. The weight of any face in a graph is the sum of labels of that particular face and its surrounding vertices and edges. For a deep survey on weights of graph elements, reader can go through [2-4]. The weight of a face $f$ of a plane graph $G$ under $k$-labeling $\phi$ of type $(\alpha, \beta, \gamma)$ can be defined as follows:

$$
\mathrm{Wt}_{\phi_{(\alpha, \beta, \gamma)}}(f)=\alpha \sum_{\nu \sim f} \phi(v)+\beta \sum_{e \sim f} \phi(e)+\gamma \phi(f) .
$$

A $k$-labeling $\phi$ of type $(\alpha, \beta, \gamma)$ of the plane graph $G$ is called face irregular $k$-labeling of type $(\alpha, \beta, \gamma)$ of the plane graph $G$ if every two different faces have distinct weights; that is, for graph faces $f, g \in G$ and $f \neq g$, we have

$$
\mathrm{Wt}_{\phi_{(\alpha, \beta, \gamma)}}(f) \neq \mathrm{Wt}_{\phi_{(\alpha, \beta, \gamma)}}(g) \text {. }
$$

Face irregularity strength of type $(\alpha, \beta, \gamma)$ of any plane graph $G$ is the minimum integer $k$ for which the graph $G$ 
admits a face irregular $k$-labeling of type $(\alpha, \beta, \gamma)$. For a vertex-edge labeled graph $G$, the minimum integer $k$ for which the graph $G$ admits a face irregular $k$-labeling of type $(\alpha, \beta, \gamma)$ is called the total face irregularity strength of type $(\alpha, \beta, \gamma)$ of the plane graph $G$, and it is denoted by $\operatorname{tfs}_{(\alpha, \beta, \gamma)}(G)$. A detailed work on irregularity strength of graphs can be seen in [4-12].

Gary Ebert et al. worked on the irregularity strength of $2 \times n$ grid in their research "Irregularity Strength for Certain Graphs," [13]. Baca et al. determined total irregularity strength of graphs and calculated bounds and exact values for different families of graphs [14]. Baca et al. investigated face irregular evaluations of plane graphs and calculated face irregularity strength of type $(\alpha, \beta, \gamma)$ for ladder graphs [15].

By motivating from all abovementioned, we are working on grid graphs $G_{n}^{m}$ with $n$ rows and $m$ columns. Labeling of a grid graph has many stages, depending on the size of graph, on the selection of rows and columns, and sometimes on the smaller and larger values of labeling. We will calculate the total face irregularity strength of grid graphs under labeling $\phi$ of type $(\alpha, \beta, \gamma)$, and this work is a modification of abovementioned articles. Grid graphs are constructed by the graph Cartesian products of path graphs, that is, $G_{n}^{m}=P_{n+1} \square P_{m+1}$.

We will prove the exact value for the total face irregularity strength under $k$-labeling $\phi$ of type $(\alpha, \beta, \gamma)$ of grid graphs with the property $\lfloor(m+1) / 3\rfloor=m-2\lfloor(m+1) / 3\rfloor$ where $1<m<n$.

We will prove the exact value for the total face irregularity strength under $k$-labeling $\phi$ of type $(\alpha, \beta, \gamma)$ of wheel graph $W_{n}$.

Baca et al. determined a lower bound for the face irregularity strength of type $(\alpha, \beta, \gamma)$ when a 2 -connected plane graph $G$ has more than one faces of the largest sizes $[14,16]$. They presented the following theorem.

Theorem 1 (see $[14,16])$. Let $G=(V, E, F)$ be a 2-connected plane graph with $n_{i} i$-sided faces, $i \geq 3$. Let $\alpha, \beta, \gamma \in\{0,1\}$, $a=\min \left\{i: n_{i} \neq 0\right\}$, and $b=\max \left\{i: n_{i} \neq 0\right\}$. Then, the face irregularity strength of type $(\alpha, \beta, \gamma)$ of the plane graph $G$ is

$$
\mathrm{fs}_{(\alpha, \beta, \gamma)}(G) \geq\left\lceil\frac{(\alpha+\beta) a+\gamma+|F(G)|-1}{(\alpha+\beta) b+\gamma}\right\rceil .
$$

Proof. Suppose that face irregularity strength under a $k$ labeling $\phi$ of type $\alpha, \beta, \gamma$ of the plane graph $G$ is $k$.

The smallest face weight under the face irregular $k$-labeling $\phi$ admits the value at least $(\alpha+\beta) a+\gamma$. Since $|F(G)|=\sum_{i=3}^{b} n_{i}$, it follows that the largest face weight attains the value at least $(\alpha+\beta) a+\gamma+|F(G)|-1$ and at most $((\alpha+\beta) b+\gamma) k$. Hence,

$$
\begin{aligned}
(\alpha+\beta) a+\gamma+|F(G)|-1 & \leq((\alpha+\beta) b+\gamma) k, \\
k & \geq\left\lceil\frac{(\alpha+\beta) a+\gamma+|F(G)|-1}{(\alpha+\beta) b+\gamma}\right\rceil .
\end{aligned}
$$

This lower bound can be improved when a 2-connected plane graph $G$ contains only one face of the largest size, that is, $n_{b}=1$ and $c=\max \left\{i: n_{i} \neq 0, i<b\right\}$. So, we present the following theorem to calculate the lower bounds for grid graphs $G_{n}^{m}$.

\section{Main Results}

In this research, we will demonstrate the tight lower bound for the total face irregular strength of type $(1 ; 1 ; 0)$ for the plan graph particularly grid and wheel graphs. It is sufficient to prove tight lower bound of grid graph that the exact value of tfs $\left(G_{m} n\right)$ exists and differences in weights of the horizontal faces must be 1 and the differences in weights of the vertical faces is $m$.

Theorem 2. Let $G=(V, E, F)$ be a 2-connected plane graph with $n_{i} i$-sided faces, $i \geq 3$. Let $\alpha, \beta, \gamma \in\{0,1\}, a=\min \left\{i: n_{i} \neq\right.$ $0\}$ and $b=\max \left\{i: n_{i} \neq 0\right\}, n_{b}=1$, and $c=\max \left\{i: n_{i} \neq 0, i\right.$ $\leq b\}$. Then, the total face irregularity strength of type $(\alpha, \beta, \gamma)$ of the plane graph $G$ is

$$
\mathrm{fs}_{(\alpha, \beta, \gamma)}(G) \geq\left\lceil\frac{(\alpha+\beta) a+\gamma+|F(G)|-2}{(\alpha+\beta) c+\gamma}\right\rceil .
$$

Proof. We suppose that total face irregularity strength of any 2 -connected plane graph $G$ under $k$-labeling $\phi$ of type $\alpha, \beta, \gamma$ is equal to $k$, that is,

$$
\operatorname{tfs}_{(\alpha, \beta, \gamma)}(G)=k .
$$

Given that the lagest face $n_{b}=1$ for $i<b$. So, the smallest face weight under the face irregular $k$-labeling $\phi$ of type $(\alpha, \beta, \gamma)$ will have the minimum value $(\alpha+\beta) c+\gamma$. The total number of faces of the graph can be obtained by adding all the number of $i$-sided faces where $i \geq 3$. Hence, the largest face weight can have the minimum value $(\alpha+\beta) a+\gamma+$ $|F(G)|-2$ and maximum value $((\alpha+\beta) c+\gamma) k$. So, we can construct the following results:

$$
\begin{gathered}
(\alpha+\beta) a+\gamma+|F(G)|-2 \leq((\alpha+\beta) b+\gamma) k \\
\Rightarrow k \geq\left\lceil\frac{(\alpha+\beta) a+\gamma+|F(G)|-2}{(\alpha+\beta) c+\gamma}\right\rceil .
\end{gathered}
$$

Hence,

$$
\operatorname{tfs}_{(\alpha, \beta, \gamma)}(G) \geq\left\lceil\frac{(\alpha+\beta) a+\gamma+|F(G)|-2}{(\alpha+\beta) c+\gamma}\right\rceil .
$$

From the above result, we see that if a 2-connected plane graph $G$ contains only one largest face, then the lower bound for the face irregularity strength of type $(1,1,0)$ can be calculated as

$$
\operatorname{tfs}_{(1,1,0)}(G) \geq\left\lceil\frac{2 a+|F(G)|-2}{2 c}\right\rceil .
$$


In this research, we will prove the tight lower bound for the total face irregularity strength of type $(1,1,0)$ for the grid graph $G_{n}^{m}$ and wheel graph $W_{n}$. To prove the tight lower bound of the grid graph, it will be sufficient to show that the exact value of tfs $\left(G_{n}^{m}\right)$ exists. The exact value of $\operatorname{tfs}\left(G_{n}^{m}\right)$, that is, calculated from grid graph $G_{n}^{m}$ under a graph $k$-labeling of type $(1,1,0)$, exists if the differences in weights of the horizontal faces are 1 and the differences in weights of the vertical faces are $m$. Generalized grid graphs can be written as $G_{n}^{m}=P_{n+1} \square P_{m+1}$.

The vertex set and the edge set of the grid graph can be defined as follows:

$$
\begin{aligned}
& V\left(P_{n+1} \square P_{m+1}\right)=\left\{v_{i}^{j}: i=1,2, \ldots, n+1, j=1,2, \ldots, m+1\right\}, \\
& E\left(P_{n+1} \square P_{m+1}\right)=\left\{v_{i}^{j} v_{i+1}^{j}: i=1,2, \ldots, n, j=1,2, \ldots, m+1\right\} \cup\left\{v_{i}^{j} v_{i}^{j+1}: i=1,2, \ldots, n+1, j=1,2, \ldots, m\right\} .
\end{aligned}
$$

Theorem 3. Let $n, m \geq 2$ be positive integers and $G_{n}^{m}=P_{n+1} \square P_{m+1}$ be generalized grid graph, then

$$
\operatorname{tfs}_{(1,1,0)}\left(P_{n+1} \square P_{m+1}\right)=\left\lceil\frac{m n+7}{8}\right\rceil .
$$

In order to prove this, it will be sufficient to show that the exact value of tfs $\left(G_{n}^{m}\right)$ exists.

The vertices for the generalized graph $G_{n}^{m}$ under a $k$ labeling $\phi$ of type $(1,1,0)$ in different intervals of $i$ and $j$ can be defined as follows:

$$
\phi\left(v_{i}^{j}\right)= \begin{cases}1+\left\lfloor\frac{m+1}{3}\right\rfloor\left\lfloor\frac{i-1}{2}\right\rfloor, & \text { for } i=1,2,3, \ldots, 2\left\lceil\frac{k}{\lfloor(m+1) / 3\rfloor}\right\rceil \text { and } j=1,2, \ldots, m+1, \\ k, & \text { for } i=2\left\lceil\frac{k}{\lfloor(m+1) / 3\rfloor}\right\rceil+1, \ldots, n+1 \text { and } j=1,2, \ldots, m+1 .\end{cases}
$$

The horizontal edges for the generalized graph $G_{n}^{m}$ under a $k$-labeling $\phi$ of type $(1,1,0)$ in different intervals of $i$ and $j$ can be defined as follows:

$$
\phi\left(v_{i}^{j} v_{i}^{j+1}\right)= \begin{cases}1+\left\lfloor\frac{m+1}{3}\right\rfloor\left\lfloor\frac{i-1}{2}\right\rfloor, & \text { for } i=1,2,3, \ldots, 2\left\lceil\frac{k}{\lfloor(m+1) / 3\rfloor}\right\rceil \text { and } j=1,2, \ldots, m, \\ k, & \text { for } i=2\left\lceil\frac{k}{\lfloor(m+1) / 3\rfloor}\right\rceil+1, \ldots, n+1 \text { and } j=1,2, \ldots, m .\end{cases}
$$

The vertical edges for the generalized graph $G_{n}^{m}$ under a $k$-labeling $\phi$ of type $(1,1,0)$ in different intervals of $i$ and $j$ can be defined as follows: 


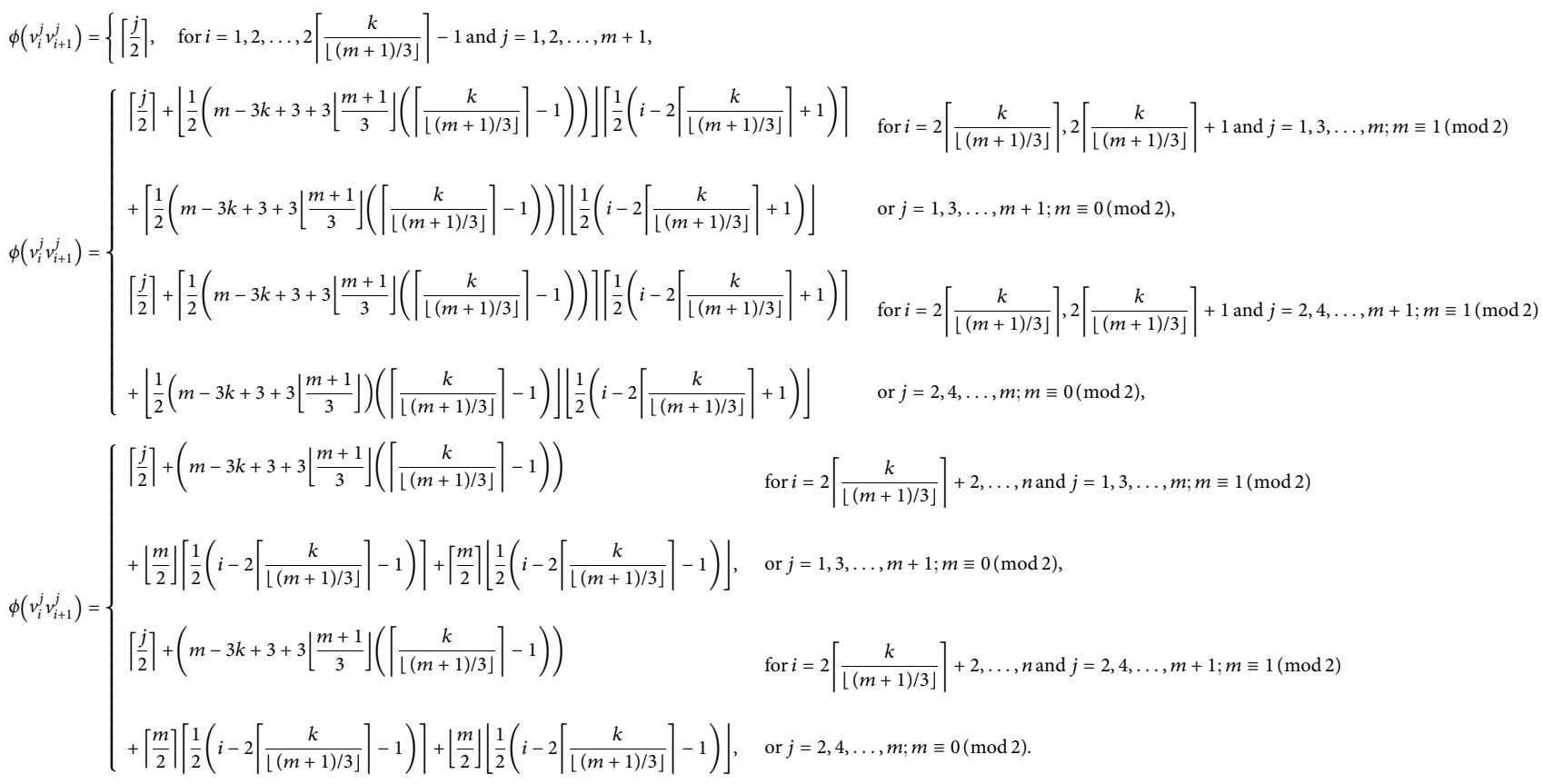

Figure 1 represents the generalized formula for face weights. The generalization of weights over the face $f$ under a $k$-labeling $\phi$ of type $(1,1,0)$ for the graph $G_{n}^{m}$ can be defined as follows:

$$
\begin{aligned}
\mathrm{Wt}_{(1,1,0)}\left(f_{i}^{j}\right)= & \sum_{v \sim f_{i}^{j}} f(v)+\sum_{e \sim f_{i}^{j}} f(e)=\phi\left(v_{i}^{j}\right)+\phi\left(v_{i}^{j+1}\right)+\phi\left(v_{i+1}^{j}\right)+\phi\left(v_{i+1}^{j+1}\right)+\phi\left(v_{i}^{j} v_{i}^{j+1}\right) \\
& +\phi\left(v_{i}^{j} v_{i+1}^{j}\right)+\phi\left(v_{i+1}^{j} v_{i+1}^{j+1}\right)+\phi\left(v_{i}^{j+1} v_{i+1}^{j+1}\right) .
\end{aligned}
$$

Horizontal differences in weights among different intervals of $i$ and $j$ can be calculated as follows:

$$
\begin{aligned}
\text { For } i= & 1,2,3, \ldots, 2\left\lceil\frac{k}{\lfloor(m+1) / 3\rfloor}\right\rceil-2 \text { and } j=1,2, \ldots, m+1, \\
\mathrm{Wt}_{(1,1,0)}\left(f_{i}^{j+1}\right)-\mathrm{Wt}_{(1,1,0)}\left(f_{i}^{j}\right)= & \phi\left(v_{i}^{j+1}\right)+\phi\left(v_{i}^{j+2}\right)+\phi\left(v_{i+1}^{j+1}\right)+\phi\left(v_{i+1}^{j+2}\right)+\phi\left(v_{i}^{j+1} v_{i}^{j+2}\right)+\phi\left(v_{i}^{j+1} v_{i+1}^{j+1}\right) \\
& +\phi\left(v_{i+1}^{j+1} v_{i+1}^{j+2}\right)+\phi\left(v_{i}^{j+2} v_{i+1}^{j+2}\right)-\phi\left(v_{i}^{j}\right)-\phi\left(v_{i}^{j+1}\right)-\phi\left(v_{i+1}^{j}\right)-\phi\left(v_{i+1}^{j+1}\right)-\phi\left(v_{i}^{j} v_{i}^{j+1}\right)-\phi\left(v_{i}^{j} v_{i+1}^{j}\right) \\
& -\phi\left(v_{i+1}^{j} v_{i+1}^{j+1}\right)-\phi\left(v_{i}^{j+1} v_{i+1}^{j+1}\right) \\
= & 1+\left\lfloor\frac{m+1}{3}\right\rfloor\left\lfloor\frac{i-1}{2}\right\rfloor+1+\left\lfloor\frac{m+1}{3}\right\rfloor\left\lfloor\frac{i}{2}\right\rfloor+1+\left\lfloor\frac{m+1}{3}\right\rfloor\left\lfloor\frac{i-1}{2}\right\rfloor+1+\left\lfloor\frac{m+1}{3}\right\rfloor\left[\frac{i}{2}\right\rfloor+\left\lfloor\frac{j+2}{2}\right\rfloor-1 \\
& -\left\lfloor\frac{m+1}{3}\right\rfloor\left\lfloor\frac{i-1}{2}\right\rfloor-1-\left\lfloor\frac{m+1}{3}\right\rfloor\left\lfloor\frac{i}{2}\right\rfloor-1-\left\lfloor\frac{m+1}{3}\right\rfloor\left[\frac{i-1}{2}\right\rfloor-\left\lceil\frac{j}{2}\right\rfloor-1-\left\lfloor\frac{m+1}{3}\right\rfloor\left\lfloor\frac{i}{2}\right\rfloor \\
= & \left\lfloor\frac{j+2}{2}\right\rceil-\left\lfloor\frac{j}{2}\right\rfloor
\end{aligned}
$$




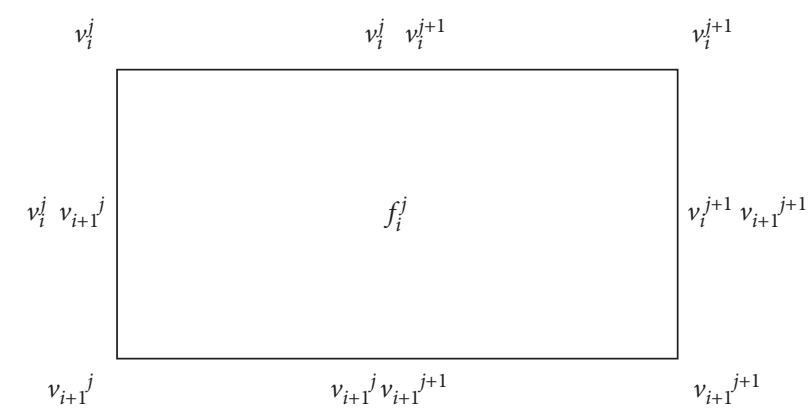

Figure 1: Construction of weights over the face $f$ under $k$-labeling of type $(1,1,0)$.

$=1$, for every value of $j$,

For $i=2\left[\frac{k}{\lfloor(m+1) / 3\rfloor}\right\rceil-1$ and $j=1,2, \ldots, m+1$,

$$
\begin{aligned}
\mathrm{Wt}_{(1,1,0)}\left(f_{i}^{j+1}\right)-\mathrm{Wt}_{(1,1,0)}\left(f_{i}^{j}\right)= & \phi\left(v_{i}^{j+1}\right)+\phi\left(v_{i}^{j+2}\right)+\phi\left(v_{i+1}^{j+1}\right)+\phi\left(v_{i+1}^{j+2}\right)+\phi\left(v_{i}^{j+1} v_{i}^{j+2}\right)+\phi\left(v_{i}^{j+1} v_{i+1}^{j+1}\right) \\
& +\phi\left(v_{i+1}^{j+1} v_{i+1}^{j+2}\right)+\phi\left(v_{i}^{j+2} v_{i+1}^{j+2}\right)-\phi\left(v_{i}^{j}\right)-\phi\left(v_{i}^{j+1}\right)-\phi\left(v_{i+1}^{j}\right)-\phi\left(v_{i+1}^{j+1}\right)-\phi\left(v_{i}^{j} v_{i}^{j+1}\right)-\phi\left(v_{i}^{j} v_{i+1}^{j}\right) \\
& -\phi\left(v_{i+1}^{j} v_{i+1}^{j+1}\right)-\phi\left(v_{i}^{j+1} v_{i+1}^{j+1}\right) \\
= & 1+\left\lfloor\frac{m+1}{3}\right\rfloor\left[\frac{i-1}{2}\right\rfloor+1+\left\lfloor\frac{m+1}{3}\right\rfloor\left\lfloor\frac{i}{2}\right\rfloor+1+\left\lfloor\frac{m+1}{3}\right\rfloor\left\lfloor\frac{i-1}{2}\right\rfloor+1+\left\lfloor\frac{m+1}{3}\right\rfloor\left\lfloor\frac{i}{2}\right\rfloor+\left\lfloor\frac{j+2}{2}\right\rceil-1 \\
& -\left\lfloor\frac{m+1}{3}\right\rfloor\left\lfloor\frac{i-1}{2}\right\rfloor-1-\left\lfloor\frac{m+1}{3}\right\rfloor\left\lfloor\frac{i}{2}\right\rfloor-1-\left\lfloor\frac{m+1}{3}\right\rfloor\left[\frac{i-1}{2}\right\rfloor-\left\lfloor\frac{j}{2}\right\rfloor-1-\left\lfloor\frac{m+1}{3}\right\rfloor\left[\frac{i}{2}\right\rfloor \\
= & \left\lceil\frac{j+2}{2}\right\rceil-\left\lfloor\frac{j}{2}\right\rfloor
\end{aligned}
$$

$=1$, for all values of $j$,

$$
\text { For } i=2\left\lceil\frac{k}{\lfloor(m+1) / 3\rfloor}\right\rceil \text { and } j=1,2, \ldots, m+1,
$$

$$
\begin{aligned}
\mathrm{Wt}_{(1,1,0)}\left(f_{i}^{j+1}\right)-\mathrm{Wt}_{(1,1,0)}\left(f_{i}^{j}\right)= & \phi\left(v_{i}^{j+1}\right)+\phi\left(v_{i}^{j+2}\right)+\phi\left(v_{i+1}^{j+1}\right)+\phi\left(v_{i+1}^{j+2}\right)+\phi\left(v_{i}^{j+1} v_{i}^{j+2}\right)+\phi\left(v_{i}^{j+1} v_{i+1}^{j+1}\right) \\
& +\phi\left(v_{i+1}^{j+1} v_{i+1}^{j+2}\right)+\phi\left(v_{i}^{j+2} v_{i+1}^{j+2}\right)-\phi\left(v_{i}^{j}\right)-\phi\left(v_{i}^{j+1}\right)-\phi\left(v_{i+1}^{j}\right)-\phi\left(v_{i+1}^{j+1}\right)-\phi\left(v_{i}^{j} v_{i}^{j+1}\right)-\phi\left(v_{i}^{j} v_{i+1}^{j}\right) \\
& -\phi\left(v_{i+1}^{j} v_{i+1}^{j+1}\right)-\phi\left(v_{i}^{j+1} v_{i+1}^{j+1}\right),
\end{aligned}
$$

$$
\begin{aligned}
& \mathrm{Wt}_{(1,1,0)}\left(f_{i}^{j+1}\right)-\mathrm{Wt}_{(1,1,0)}\left(f_{i}^{j}\right)=1+\left\lfloor\frac{m+1}{3}\right\rfloor\left\lfloor\frac{i-1}{2}\right\rfloor+k+1+\left\lfloor\frac{m+1}{3}\right\rfloor\left\lfloor\frac{i-1}{2}\right\rfloor+k+\left\lceil\frac{j+2}{2}\right\rceil \\
& +\left\lfloor\frac{1}{2}\left(m-3 k+3+3\left\lfloor\frac{m+1}{3}\right\rfloor\right)\left(\left\lceil\frac{k}{\lfloor(m+1) / 3\rfloor}\right\rceil-1\right)\right\rfloor\left|\frac{1}{2}\left(i+1-2\left\lceil\frac{k}{\lfloor(m+1) / 3\rfloor}\right\rceil+1\right)\right| \\
& +\left\lceil\frac{1}{2}\left(m-3 k+3+3\left\lfloor\frac{m+1}{3}\right\rfloor\left(\left\lceil\frac{k}{\lfloor(m+1) / 3\rfloor}\right\rceil-1\right)\right)\right\rceil\left\lfloor\frac{1}{2}\left(i+1-2\left\lceil\frac{k}{\lfloor(m+1) / 3\rfloor}\right\rceil+1\right)\right\rfloor \\
& -1-\left\lfloor\frac{m+1}{3}\right\rfloor\left\lfloor\frac{i-1}{2}\right\rfloor-k-1-\left\lfloor\frac{m+1}{3}\right\rfloor\left\lfloor\frac{i-1}{2}\right\rfloor-\left\lceil\frac{j}{2}\right\rfloor \\
& -\left\lfloor\frac{1}{2}\left(m-3 k+3+3\left\lfloor\frac{m+1}{3}\right\rfloor\right)\left(\left\lceil\frac{k}{\lfloor(m+1) / 3\rfloor}\right\rceil-1\right)\right\rfloor\left[\frac{1}{2}\left(i-2\left\lceil\frac{k}{\lfloor(m+1) / 3\rfloor}\right\rceil+1\right)\right\rceil
\end{aligned}
$$




$$
\begin{aligned}
& -\left\lceil\frac{1}{2}\left(m-3 k+3+3\left\lfloor\frac{m+1}{3}\right\rfloor\left(\left\lceil\frac{k}{\lfloor(m+1) / 3\rfloor}\right]-1\right)\right) \mid\left\lfloor\frac{1}{2}\left(i-2\left\lceil\frac{k}{\lfloor(m+1) / 3\rfloor}\right]+1\right)\right\rfloor-k\right. \\
= & \left\lceil\frac{j+2}{2}\right\rceil+\left\lfloor\frac{1}{2}\left(m-3 k+3+3\left\lfloor\frac{m+1}{3}\right\rfloor\right)\left(\left\lceil\frac{k}{\lfloor(m+1) / 3\rfloor}\right]-1\right)\right\rfloor(1)+0-\left\lceil\frac{j}{2}\right\rfloor \\
& -\left\lfloor\frac{1}{2}\left(m-3 k+3+3\left\lfloor\frac{m+1}{3}\right\rfloor\right)\left(\left\lfloor\frac{k}{\lfloor(m+1) / 3\rfloor}\right]-1\right)\right\rfloor(1)-0 \\
= & \left\lceil\frac{j+2}{2}\right\rceil-\left\lceil\frac{j}{2}\right\rceil \\
= & 1, \quad \text { for all } j=1,2, \ldots, m+1,
\end{aligned}
$$$$
\text { For } i=2\left\lceil\frac{k}{\lfloor(m+1) / 3\rfloor}\right\rceil+1 \text { and } j=1,3, \ldots, m ; m \equiv 1(\bmod 2) \text { or } j=1,3, \ldots, m+1 ; m \equiv 0(\bmod 2) \text {, }
$$

$$
\begin{aligned}
\mathrm{Wt}_{(1,1,0)}\left(f_{i}^{j+1}\right)-\mathrm{Wt}_{(1,1,0)}\left(f_{i}^{j}\right)= & \phi\left(v_{i}^{j+1}\right)+\phi\left(v_{i}^{j+2}\right)+\phi\left(v_{i+1}^{j+1}\right)+\phi\left(v_{i+1}^{j+2}\right)+\phi\left(v_{i}^{j+1} v_{i}^{j+2}\right)+\phi\left(v_{i}^{j+1} v_{i+1}^{j+1}\right) \\
& +\phi\left(v_{i+1}^{j+1} v_{i+1}^{j+2}\right)+\phi\left(v_{i}^{j+2} v_{i+1}^{j+2}\right)-\phi\left(v_{i}^{j}\right)-\phi\left(v_{i}^{j+1}\right)-\phi\left(v_{i+1}^{j}\right)-\phi\left(v_{i+1}^{j+1}\right)-\phi\left(v_{i}^{j} v_{i}^{j+1}\right)-\phi\left(v_{i}^{j} v_{i+1}^{j}\right) \\
& -\phi\left(v_{i+1}^{j} v_{i+1}^{j+1}\right)-\phi\left(v_{i}^{j+1} v_{i+1}^{j+1}\right)
\end{aligned}
$$$$
\mathrm{Wt}_{(1,1,0)}\left(f_{i}^{j+1}\right)-\mathrm{Wt}_{(1,1,0)}\left(f_{i}^{j}\right)=k+k+k+k+\left\lceil\frac{j+2}{2}\right\rceil
$$$$
+\left\lfloor\frac{1}{2}\left(m-3 k+3+3\left\lfloor\frac{m+1}{3}\right\rfloor\right)\left(\left\lceil\frac{k}{\lfloor(m+1) / 3\rfloor}\right]-1\right)\right\rfloor\left\lceil\frac{1}{2}\left(i-2\left\lceil\frac{k}{\lfloor(m+1) / 3\rfloor}\right\rceil+1\right)\right\rceil
$$$$
\left.+\left\lceil\frac{1}{2}\left(m-3 k+3+3\left\lfloor\frac{m+1}{3}\right\rfloor\left(\left\lceil\frac{k}{\lfloor(m+1) / 3\rfloor}\right\rceil-1\right)\right)\right\rceil \frac{1}{2}\left(i-2\left\lceil\frac{k}{\lfloor(m+1) / 3\rfloor}\right\rceil+1\right)\right\rfloor
$$$$
-k-k-k-k-\left\lceil\frac{j}{2}\right\rceil
$$$$
-\left\lfloor\frac{1}{2}\left(m-3 k+3+3\left\lfloor\frac{m+1}{3}\right\rfloor\right)\left(\left\lceil\frac{k}{\lfloor(m+1) / 3\rfloor}\right\rceil-1\right)\right\rfloor\left\lceil\frac{1}{2}\left(i-2\left\lceil\frac{k}{\lfloor(m+1) / 3\rfloor}\right\rceil+1\right)\right\rceil
$$$$
\left.-\left\lceil\frac{1}{2}\left(m-3 k+3+3\left\lfloor\frac{m+1}{3}\right\rfloor\left(\left\lceil\frac{k}{\lfloor(m+1) / 3\rfloor}\right\rceil-1\right)\right)\right\rceil \frac{1}{2}\left(i-2\left\lceil\frac{k}{\lfloor(m+1) / 3\rfloor}\right\rceil+1\right)\right\rfloor
$$$$
=\left\lceil\frac{j+2}{2}\right\rceil-\left\lceil\frac{j}{2}\right\rceil
$$

$=1$, for every odd value of $j$,

$$
\text { For } i=2\left[\frac{k}{\lfloor(m+1) / 3\rfloor}\right\rceil+1 \text { and } j=2,4, \ldots, m+1 ; m \equiv 1(\bmod 2) \text { or } j=2,4, \ldots, m ; m \equiv 0(\bmod 2) \text {, }
$$

$$
\begin{aligned}
\mathrm{Wt}_{(1,1,0)}\left(f_{i}^{j+1}\right)-\mathrm{Wt}_{(1,1,0)}\left(f_{i}^{j}\right)= & \phi\left(v_{i}^{j+1}\right)+\phi\left(v_{i}^{j+2}\right)+\phi\left(v_{i+1}^{j+1}\right)+\phi\left(v_{i+1}^{j+2}\right)+\phi\left(v_{i}^{j+1} v_{i}^{j+2}\right)+\phi\left(v_{i}^{j+1} v_{i+1}^{j+1}\right) \\
& +\phi\left(v_{i+1}^{j+1} v_{i+1}^{j+2}\right)+\phi\left(v_{i}^{j+2} v_{i+1}^{j+2}\right)-\phi\left(v_{i}^{j}\right)-\phi\left(v_{i}^{j+1}\right)-\phi\left(v_{i+1}^{j}\right)-\phi\left(v_{i+1}^{j+1}\right)-\phi\left(v_{i}^{j} v_{i}^{j+1}\right)-\phi\left(v_{i}^{j} v_{i+1}^{j}\right) \\
& -\phi\left(v_{i+1}^{j} v_{i+1}^{j+1}\right)-\phi\left(v_{i}^{j+1} v_{i+1}^{j+1}\right)
\end{aligned}
$$

$\mathrm{Wt}_{(1,1,0)}\left(f_{i}^{j+1}\right)-\mathrm{Wt}_{(1,1,0)}\left(f_{i}^{j}\right)=k+k+k+k+\left\lceil\frac{j+2}{2}\right\rceil$ 


$$
\begin{aligned}
& +\left\lceil\frac{1}{2}\left(m-3 k+3+3\left\lfloor\frac{m+1}{3}\right\rfloor\right)\left(\left\lceil\frac{k}{\lfloor(m+1) / 3\rfloor}\right]-1\right)|| \frac{1}{2}\left(i-2\left\lceil\frac{k}{\lfloor(m+1) / 3\rfloor}\right\rceil+1\right) \mid\right. \\
& \left.+\left\lfloor\frac{1}{2}\left(m-3 k+3+3\left\lfloor\frac{m+1}{3}\right\rfloor\right)\left(\left\lceil\frac{k}{\lfloor(m+1) / 3\rfloor}\right]-1\right)\right] \mid \frac{1}{2}\left(i-2\left\lceil\frac{k}{\lfloor(m+1) / 3\rfloor}\right\rfloor+1\right)\right] \\
& -k-k-k-k-\left\lceil\frac{j}{2}\right\rfloor \\
& -\left\lceil\frac{1}{2}\left(m-3 k+3+3\left\lfloor\frac{m+1}{3}\right\rfloor\right)\left(\left\lceil\frac{k}{\lfloor(m+1) / 3\rfloor}\right]-1\right)|| \frac{1}{2}\left(i-2\left\lceil\frac{k}{\lfloor(m+1) / 3\rfloor}\right\rceil+1\right) \mid\right. \\
& -\left\lfloor\frac{1}{2}\left(m-3 k+3+3\left\lfloor\frac{m+1}{3}\right\rfloor\right)\left(\left\lceil\frac{k}{\lfloor(m+1) / 3\rfloor}\right]-1\right)|| \frac{1}{2}\left(i-2\left\lceil\frac{k}{\lfloor(m+1) / 3\rfloor}\right\rceil+1\right)\right] \\
& =\left\lceil\frac{j+2}{2}\right\rceil-\left\lceil\frac{j}{2}\right\rceil \\
& =1, \quad \text { for every even value of } j,
\end{aligned}
$$

For $i=2\left[\frac{k}{\lfloor(m+1) / 3\rfloor}\right]+2, \ldots, n ; j=1,3, \ldots, m ; m \equiv 1(\bmod 2)$ OR $j=1,3, \ldots, m+1 ; m \equiv 0(\bmod 2)$,

$$
\begin{aligned}
\mathrm{Wt}_{(1,1,0)}\left(f_{i}^{j+1}\right)-\mathrm{Wt}_{(1,1,0)}\left(f_{i}^{j}\right)= & \phi\left(v_{i}^{j+1}\right)+\phi\left(v_{i}^{j+2}\right)+\phi\left(v_{i+1}^{j+1}\right)+\phi\left(v_{i+1}^{j+2}\right)+\phi\left(v_{i}^{j+1} v_{i}^{j+2}\right)+\phi\left(v_{i}^{j+1} v_{i+1}^{j+1}\right) \\
& +\phi\left(v_{i+1}^{j+1} v_{i+1}^{j+2}\right)+\phi\left(v_{i}^{j+2} v_{i+1}^{j+2}\right)-\phi\left(v_{i}^{j}\right)-\phi\left(v_{i}^{j+1}\right)-\phi\left(v_{i+1}^{j}\right)-\phi\left(v_{i+1}^{j+1}\right)-\phi\left(v_{i}^{j} v_{i}^{j+1}\right)-\phi\left(v_{i}^{j} v_{i+1}^{j}\right) \\
& -\phi\left(v_{i+1}^{j} v_{i+1}^{j+1}\right)-\phi\left(v_{i}^{j+1} v_{i+1}^{j+1}\right),
\end{aligned}
$$$$
\mathrm{Wt}_{(1,1,0)}\left(f_{i}^{j+1}\right)-\mathrm{Wt}_{(1,1,0)}\left(f_{i}^{j}\right)=k+k+k+k+\left\lceil\frac{j+2}{2}\right\rceil+\left(m-3 k+3+3\left\lfloor\frac{m+1}{3}\right\rfloor\left(\left\lceil\frac{k}{\lfloor(m+1) / 3\rfloor}\right\rceil-1\right)\right)
$$$$
+\left\lfloor\frac{m}{2}\right\rfloor\left\lceil\frac{1}{2}\left(i-2\left\lceil\frac{k}{\lfloor(m+1) / 3\rfloor}\right\rceil-1\right)\right\rceil+\left\lceil\frac{m}{2}\right\rceil\left\lfloor\frac{1}{2}\left(i-2\left\lceil\frac{k}{\lfloor(m+1) / 3\rfloor}\right\rceil-1\right)\right\rfloor-k-k-k-k-\left\lceil\frac{j}{2}\right\rceil
$$$$
-\left(m-3 k+3+3\left\lfloor\frac{m+1}{3}\right\rfloor\left(\left\lceil\frac{k}{\lfloor(m+1) / 3\rfloor}\right\rceil-1\right)\right)-\left\lfloor\frac{m}{2}\right\rfloor\left\lceil\frac{1}{2}\left(i-2\left\lceil\frac{k}{\lfloor(m+1) / 3\rfloor}\right\rceil-1\right)\right\rceil
$$$$
-\left\lceil\frac{m}{2}\right\rceil\left\lfloor\frac{1}{2}\left(i-2\left\lceil\frac{k}{\lfloor(m+1) / 3\rfloor}\right\rceil-1\right)\right\rfloor
$$$$
=\left\lceil\frac{j+2}{2}\right\rceil-\left\lceil\frac{j}{2}\right\rceil
$$

$=1$, for all odd values of $j$,

$$
\text { For } i=2\left\lceil\frac{k}{\lfloor(m+1) / 3\rfloor}\right\rceil+2, \ldots, n ; j=2,4, \ldots, m+1 ; m \equiv 1(\bmod 2) \text { OR } j=2,4, \ldots, m ; m \equiv 0(\bmod 2),
$$

$$
\begin{aligned}
\mathrm{Wt}_{(1,1,0)}\left(f_{i}^{j+1}\right)-\mathrm{Wt}_{(1,1,0)}\left(f_{i}^{j}\right)= & \phi\left(v_{i}^{j+1}\right)+\phi\left(v_{i}^{j+2}\right)+\phi\left(v_{i+1}^{j+1}\right)+\phi\left(v_{i+1}^{j+2}\right)+\phi\left(v_{i}^{j+1} v_{i}^{j+2}\right)+\phi\left(v_{i}^{j+1} v_{i+1}^{j+1}\right) \\
& +\phi\left(v_{i+1}^{j+1} v_{i+1}^{j+2}\right)+\phi\left(v_{i}^{j+2} v_{i+1}^{j+2}\right)-\phi\left(v_{i}^{j}\right)-\phi\left(v_{i}^{j+1}\right)-\phi\left(v_{i+1}^{j}\right)-\phi\left(v_{i+1}^{j+1}\right)-\phi\left(v_{i}^{j} v_{i}^{j+1}\right)-\phi\left(v_{i}^{j} v_{i+1}^{j}\right) \\
& -\phi\left(v_{i+1}^{j} v_{i+1}^{j+1}\right)-\phi\left(v_{i}^{j+1} v_{i+1}^{j+1}\right),
\end{aligned}
$$

$\mathrm{Wt}_{(1,1,0)}\left(f_{i}^{j+1}\right)-\mathrm{Wt}_{(1,1,0)}\left(f_{i}^{j}\right)=k+k+k+k+\left\lceil\frac{j+2}{2}\right\rceil+\left(m-3 k+3+3\left\lfloor\frac{m+1}{3}\right\rfloor\left(\left\lceil\frac{k}{\lfloor(m+1) / 3\rfloor}\right\rceil-1\right)\right)$ 


$$
\begin{aligned}
& +\left\lceil\frac { m } { 2 } \left\lceil\left\lceil\frac{1}{2}\left(i-2\left\lceil\frac{k}{\lfloor(m+1) / 3\rfloor}\right\rceil-1\right)\right\rceil+\left\lfloor\frac{m}{2}\right\rfloor\left\lfloor\frac{1}{2}\left(i-2\left\lceil\frac{k}{\lfloor(m+1) / 3\rfloor}\right\rceil-1\right)\right\rfloor-k-k-k-k-\left\lceil\frac{j}{2}\right\rceil\right.\right. \\
& -\left(m-3 k+3+3\left\lfloor\frac{m+1}{3}\right\rfloor\left(\left\lceil\frac{k}{\lfloor(m+1) / 3\rfloor}\right\rceil-1\right)\right)-\left\lceil\frac { m } { 2 } \left\lceil\left\lceil\frac{1}{2}\left(i-2\left\lceil\frac{k}{\lfloor(m+1) / 3\rfloor}\right\rceil-1\right)\right]\right.\right. \\
& -\left\lfloor\frac{m}{2}\right\rfloor\left\lfloor\frac{1}{2}\left(i-2\left\lceil\frac{k}{\lfloor(m+1) / 3\rfloor}\right\rceil-1\right)\right\rfloor \\
& =\left\lceil\frac{j+2}{2}\right\rceil-\left\lceil\frac{j}{2}\right\rfloor
\end{aligned}
$$

$=1, \quad$ for all even values of $j$.

Vertical differences in weights among different intervals of $i$ and $j$ can be calculated as follows:

$$
\text { For } i=1,2, \ldots, 2\left\lceil\frac{k}{\lfloor(m+1) / 3\rfloor}\right\rceil-2 \text { and } j=1,2, \ldots, m+1 \text {, }
$$

$$
\begin{aligned}
\mathrm{Wt}_{(1,1,0)}\left(f_{i+1}^{j}\right)-\mathrm{Wt}_{(1,1,0)}\left(f_{i}^{j}\right)= & \phi\left(v_{i+1}^{j}\right)+\phi\left(v_{i+1}^{j+1}\right)+\phi\left(v_{i+2}^{j}\right)+\phi\left(v_{i+2}^{j+1}\right)+\phi\left(v_{i+1}^{j} v_{i+1}^{j+1}\right)+\phi\left(v_{i+1}^{j} v_{i+2}^{j}\right) \\
& +\phi\left(v_{i+2}^{j} v_{i+2}^{j+1}\right)+\phi\left(v_{i+1}^{j+1} v_{i+2}^{j+1}\right)-\phi\left(v_{i}^{j}\right)-\phi\left(v_{i}^{j+1}\right)-\phi\left(v_{i+1}^{j}\right)-\phi\left(v_{i+1}^{j+1}\right)-\phi\left(v_{i}^{j} v_{i}^{j+1}\right)-\phi\left(v_{i}^{j} v_{i+1}^{j}\right) \\
& -\phi\left(v_{i+1}^{j} v_{i+1}^{j+1}\right)-\phi\left(v_{i}^{j+1} v_{i+1}^{j+1}\right),
\end{aligned}
$$$$
\mathrm{Wt}_{(1,1,0)}\left(f_{i+1}^{j}\right)-\mathrm{Wt}_{(1,1,0)}\left(f_{i}^{j}\right)=1+\left\lfloor\frac{m+1}{3}\right\rfloor\left\lfloor\frac{i+1}{2}\right\rfloor+1+\left\lfloor\frac{m+1}{3}\right\rfloor\left\lfloor\frac{i+1}{2}\right\rfloor+\left\lceil\frac{j}{2}\right\rceil+\left\lceil\frac{j+1}{2}\right\rceil+1
$$$$
+\left\lfloor\frac{m+1}{3}\right\rfloor\left\lfloor\frac{i+1}{2}\right\rfloor-1-\left\lfloor\frac{m+1}{3}\right\rfloor\left\lfloor\frac{i-1}{2}\right\rfloor-1-\left\lfloor\frac{m+1}{3}\right\rfloor\left\lfloor\frac{i-1}{2}\right\rfloor-1-\left\lfloor\frac{m+1}{3}\right\rfloor\left\lfloor\frac{i-1}{2}\right\rfloor
$$$$
-\left\lceil\frac{j}{2}\right\rceil-\left\lceil\frac{j+1}{2}\right\rceil
$$$$
=3\left\lfloor\frac{m+1}{3}\right\rfloor\left\lfloor\frac{i+1}{2}\right\rfloor-3\left\lfloor\frac{m+1}{3}\right\rfloor\left\lfloor\frac{i-1}{2}\right\rfloor
$$$$
=3\left\lfloor\frac{m+1}{3}\right\rfloor\left(\left\lfloor\frac{i+1}{2}\right\rfloor-\left\lfloor\frac{i-1}{2}\right\rfloor\right)
$$$$
=3\left\lfloor\frac{m+1}{3}\right\rfloor(1)
$$$$
=m \text {, }
$$

$$
\text { For } i=2\left\lceil\frac{k}{\lfloor(m+1) / 3\rfloor}\right\rceil-1 ; j=1,2, \ldots, m+1,
$$

$$
\begin{aligned}
\mathrm{Wt}_{(1,1,0)}\left(f_{i+1}^{j}\right)-\mathrm{Wt}_{(1,1,0)}\left(f_{i}^{j}\right)= & \phi\left(v_{i+1}^{j}\right)+\phi\left(v_{i+1}^{j+1}\right)+\phi\left(v_{i+2}^{j}\right)+\phi\left(v_{i+2}^{j+1}\right)+\phi\left(v_{i+1}^{j} v_{i+1}^{j+1}\right)+\phi\left(v_{i+1}^{j} v_{i+2}^{j}\right) \\
& +\phi\left(v_{i+2}^{j} v_{i+2}^{j+1}\right)+\phi\left(v_{i+1}^{j+1} v_{i+2}^{j+1}\right)-\phi\left(v_{i}^{j}\right)-\phi\left(v_{i}^{j+1}\right)-\phi\left(v_{i+1}^{j}\right)-\phi\left(v_{i+1}^{j+1}\right)-\phi\left(v_{i}^{j} v_{i}^{j+1}\right)-\phi\left(v_{i}^{j} v_{i+1}^{j}\right)
\end{aligned}
$$


Journal of Mathematics

9

$$
\begin{aligned}
& -\phi\left(v_{i+1}^{j} l_{i+1}^{j+1}\right)-\phi\left(v_{i}^{j+1} v_{i+1}^{j+1}\right), \\
& \mathrm{Wt}_{(1,1,0)}\left(f_{i+1}^{j}\right)-\mathrm{Wt}_{(1,1,0)}\left(f_{i}^{j}\right)=k+k+k+\left\lceil\frac{j}{2}\right\rceil+\left\lceil\frac{j+1}{2}\right\rceil \\
& +\left\lceil\frac{1}{2}\left(i+1-2\left\lceil\frac{k}{\lfloor(m+1) / 3\rfloor}\right\rceil+1\right)\right\rceil \\
& \cdot\left(\left\lfloor\frac{1}{2}\left(m-3 k+3+3\left\lfloor\frac{m+1}{3}\right\rfloor\left(\left\lceil\frac{k}{\lfloor(m+1) / 3\rfloor}\right\rceil-1\right)\right)\right\rfloor\right. \\
& \left.+\left\lceil\frac{1}{2}\left(m-3 k+3+3\left[\frac{m+1}{3}\right\rfloor\left(\left\lceil\frac{k}{\lfloor(m+1) / 3\rfloor}\right\rceil-1\right)\right)\right\rceil\right) \\
& +\left\lfloor\frac{1}{2}\left(i+1-2\left\lceil\frac{k}{\lfloor(m+1) / 3\rfloor}\right\rceil+1\right)\right\rfloor \\
& \cdot\left(\left\lceil\frac{1}{2}\left(m-3 k+3+3\left\lfloor\frac{m+1}{3}\right\rfloor\left(\left\lceil\frac{k}{\lfloor(m+1) / 3\rfloor}\right\rceil-1\right)\right)\right)\right. \\
& \left.+\left\lfloor\frac{1}{2}\left(m-3 k+3+3\left\lfloor\frac{m+1}{3}\right\rfloor\left(\left[\frac{k}{\lfloor(m+1) / 3\rfloor}\right\rceil-1\right)\right)\right\rfloor\right) \\
& -1-\left\lfloor\frac{m+1}{3}\right\rfloor\left\lfloor\frac{i-1}{2}\right\rfloor-1-\left\lfloor\frac{m+1}{3}\right\rfloor\left\lfloor\frac{i-1}{2}\right\rfloor-1-\left\lfloor\frac{m+1}{3}\right\rfloor\left\lfloor\frac{i-1}{2}\right\rfloor-\left\lceil\frac{j}{2}\right\rceil-\left\lceil\frac{j+1}{2}\right\rceil \\
& =3 k+\left\lceil\frac{1}{2}\left(i+1-2\left\lceil\frac{k}{\lfloor(m+1) / 3\rfloor}\right\rceil+1\right)\right\rceil \text {, } \\
& \cdot\left(\left\lfloor\frac{(m-3 k+3+3\lfloor(m+1) / 3\rfloor)(\lceil k /\lfloor(m+1) / 3\rfloor\rceil-1)}{2}\right\rfloor\right. \\
& \left.+\left\lceil\frac{(m-3 k+3+3\lfloor(m+1) / 3\rfloor(\lceil k /\lfloor(m+1) / 3\rfloor\rceil-1))}{2}\right\rceil\right) \\
& +\left\lfloor\frac{1}{2}(i+1-2\lceil k /\lfloor(m+1) / 3\rfloor\rceil+1)\right\rfloor \\
& \cdot\left(\left\lceil\frac{(m-3 k+3+3\lfloor(m+1) / 3\rfloor(\lceil k /\lfloor(m+1) / 3\rfloor\rceil-1))}{2}\right\rceil\right. \\
& \left.+\left\lfloor\frac{(m-3 k+3+3\lfloor(m+1) / 3\rfloor(\lceil k /\lfloor(m+1) / 3\rfloor\rceil-1))}{2}\right\rfloor\right) \\
& -3-3\left\lfloor\frac{m+1}{3}\right\rfloor\left\lfloor\frac{i-1}{2}\right\rfloor \\
& =3 k+(1)\left(m-3 k+3+3\left\lfloor\frac{m+1}{3}\right\rfloor\left(\left\lceil\frac{k}{\lfloor(m+1) / 3\rfloor}\right\rfloor-1\right)\right)+0-3-3\left\lfloor\frac{m+1}{3}\right\rfloor\left\lfloor\frac{i-1}{2}\right\rfloor \\
& =3 k+m-3 k+3+3\left\lfloor\frac{m+1}{3}\right\rfloor\left\lceil\frac{k}{\lfloor(m+1) / 3\rfloor}\right]-3\left\lfloor\frac{m+1}{3}\right\rfloor-3-3\left\lfloor\frac{m+1}{3}\right\rfloor\left\lfloor\frac{i-1}{2}\right\rfloor \\
& =m+3\left\lfloor\frac{m+1}{3}\right\rfloor\left(\left\lceil\frac{k}{\lfloor(m+1) / 3\rfloor}\right\rfloor-1-\left\lfloor\frac{i-1}{2}\right\rfloor\right)
\end{aligned}
$$


10

Journal of Mathematics

$$
\begin{aligned}
& =m+3\left\lfloor\frac{m+1}{3}\right\rfloor\left(\left\lceil\frac{k}{\lfloor(m+1) / 3\rfloor}\right\rfloor-1-\left\lfloor\left\lceil\frac{k}{\lfloor(m+1) / 3\rfloor}\right\rceil-1\right\rfloor\right) \\
& =m \text {, } \\
& \text { For } i=2\left\lceil\frac{k}{\lfloor(m+1) / 3\rfloor}\right\rceil ; j=1,2, \ldots, m+1, \\
& \mathrm{Wt}_{(1,1,0)}\left(f_{i+1}^{j}\right)-\mathrm{Wt}_{(1,1,0)}\left(f_{i}^{j}\right)=\phi\left(v_{i+1}^{j}\right)+\phi\left(v_{i+1}^{j+1}\right)+\phi\left(v_{i+2}^{j}\right)+\phi\left(v_{i+2}^{j+1}\right)+\phi\left(v_{i+1}^{j} v_{i+1}^{j+1}\right)+\phi\left(v_{i+1}^{j} v_{i+2}^{j}\right) \\
& +\phi\left(v_{i+2}^{j} v_{i+2}^{j+1}\right)+\phi\left(v_{i+1}^{j+1} v_{i+2}^{j+1}\right)-\phi\left(v_{i}^{j}\right)-\phi\left(v_{i}^{j+1}\right)-\phi\left(v_{i+1}^{j}\right)-\phi\left(v_{i+1}^{j+1}\right)-\phi\left(v_{i}^{j} v_{i}^{j+1}\right)-\phi\left(v_{i}^{j} v_{i+1}^{j}\right) \\
& -\phi\left(v_{i+1}^{j} v_{i+1}^{j+1}\right)-\phi\left(v_{i}^{j+1} v_{i+1}^{j+1}\right), \\
& \mathrm{Wt}_{(1,1,0)}\left(f_{i+1}^{j}\right)-\mathrm{Wt}_{(1,1,0)}\left(f_{i}^{j}\right)=k+k+k+\left\lceil\frac{j}{2}\right\rceil+\left\lceil\frac{j+1}{2}\right\rceil \\
& +\left\lceil\frac{1}{2}\left(i+1-2\left\lceil\frac{k}{\lfloor(m+1) / 3\rfloor}\right\rceil+1\right)\right\rceil \\
& \cdot\left(\left\lfloor\frac{1}{2}\left(m-3 k+3+3\left\lfloor\frac{m+1}{3}\right\rfloor\left(\left\lfloor\frac{k}{\lfloor(m+1) / 3\rfloor}\right\rfloor-1\right)\right)\right\rfloor\right. \\
& \left.+\left\lceil\frac{1}{2}\left(m-3 k+3+3\left\lfloor\frac{m+1}{3}\right\rfloor\left(\left\lceil\frac{k}{\lfloor(m+1) / 3\rfloor}\right\rceil-1\right)\right)\right\rceil\right) \\
& +\left\lfloor\frac{1}{2}\left(i+1-2\left\lceil\frac{k}{\lfloor(m+1) / 3\rfloor}\right\rceil+1\right)\right\rfloor \\
& \cdot\left(\left\lceil\frac{1}{2}\left(m-3 k+3+3\left\lfloor\frac{m+1}{3}\right\rfloor\left(\left\lceil\frac{k}{\lfloor(m+1) / 3\rfloor}\right\rceil-1\right)\right)\right\rceil\right. \\
& \left.+\left\lfloor\frac{1}{2}\left(m-3 k+3+3\left\lfloor\frac{m+1}{3}\right\rfloor\left(\left\lfloor\frac{k}{\lfloor(m+1) / 3\rfloor}\right\rceil-1\right)\right)\right\rfloor\right) \\
& -1-\left\lfloor\frac{m+1}{3}\right\rfloor\left\lfloor\frac{i-1}{2}\right\rfloor-1-\left\lfloor\frac{m+1}{3}\right\rfloor\left\lfloor\frac{i-1}{2}\right\rfloor-1-\left\lfloor\frac{m+1}{3}\right\rfloor\left\lfloor\frac{i-1}{2}\right\rfloor-\left\lceil\frac{j}{2}\right\rceil-\left\lceil\frac{j+1}{2}\right\rceil \\
& -\left\lceil\frac{1}{2}\left(i-2\left\lceil\frac{k}{\lfloor(m+1) / 3\rfloor}\right\rceil+1\right)\right\rceil \\
& \cdot\left(\left\lfloor\frac{1}{2}\left(m-3 k+3+3\left\lfloor\frac{m+1}{3}\right\rfloor\left(\left\lfloor\frac{k}{\lfloor(m+1) / 3\rfloor}\right\rfloor-1\right)\right)\right\rfloor\right. \\
& \left.+\left\lceil\frac{1}{2}\left(m-3 k+3+3\left[\frac{m+1}{3}\right\rfloor\left(\left[\frac{k}{\lfloor(m+1) / 3\rfloor}\right\rceil-1\right)\right)\right]\right), \\
& -\left\lfloor\frac{1}{2}\left(i-2\left\lceil\frac{k}{\lfloor(m+1) / 3\rfloor}\right\rceil+1\right)\right\rfloor \\
& \cdot\left(\left\lceil\frac{1}{2}\left(m-3 k+3+3\left\lfloor\frac{m+1}{3}\right\rfloor\left(\left\lceil\frac{k}{\lfloor(m+1) / 3\rfloor}\right\rceil-1\right)\right)\right\rceil\right. \\
& \left.+\left\lfloor\frac{1}{2}\left(m-3 k+3+3\left\lfloor\frac{m+1}{3}\right\rfloor\left(\left\lfloor\frac{k}{\lfloor(m+1) / 3\rfloor}\right\rfloor-1\right)\right)\right\rfloor\right)
\end{aligned}
$$




$$
\begin{aligned}
& =3 k \\
& +\left(\left\lfloor\frac{1}{2}\left(m-3 k+3+3\left\lfloor\frac{m+1}{3}\right\rfloor\left(\left\lfloor\frac{k}{\lfloor(m+1) / 3\rfloor}\right\rceil-1\right)\right)\right\rfloor\right. \\
& \left.+\left\lceil\frac{1}{2}\left(m-3 k+3+3\left[\frac{m+1}{3}\right\rfloor\left(\left[\frac{k}{\lfloor(m+1) / 3\rfloor}\right]-1\right)\right)\right]\right) \\
& +\left(\left\lceil\frac{1}{2}\left(m-3 k+3+3\left\lfloor\frac{m+1}{3}\right\rfloor\left(\left\lceil\frac{k}{\lfloor(m+1) / 3\rfloor}\right\rceil-1\right)\right)\right]\right. \\
& \left.+\left\lfloor\frac{1}{2}\left(m-3 k+3+3\left\lfloor\frac{m+1}{3}\right\rfloor\left(\left\lfloor\frac{k}{\lfloor(m+1) / 3\rfloor}\right\rfloor-1\right)\right)\right\rfloor\right) \\
& -3-3\left\lfloor\frac{m+1}{3}\right\rfloor\left\lfloor\frac{i-1}{2}\right\rfloor \\
& -\left(\left\lfloor\frac{1}{2}\left(m-3 k+3+3\left\lfloor\frac{m+1}{3}\right\rfloor\left(\left\lfloor\frac{k}{\lfloor(m+1) / 3\rfloor}\right\rceil-1\right)\right)\right\rfloor\right. \\
& \left.+\left\lceil\frac{1}{2}\left(m-3 k+3+3\left[\frac{m+1}{3}\right]\left(\left[\frac{k}{\lfloor(m+1) / 3\rfloor}\right]-1\right)\right)\right]\right) \\
& =3 k+\left(\left\lceil\frac{(m-3 k+3+3\lfloor m+1 / 3\rfloor(\lceil k /\lfloor(m+1) / 3\rfloor\rceil-1))}{2}\right\rceil\right. \\
& \left.+\left\lfloor\frac{(m-3 k+3+3\lfloor m+1 / 3\rfloor(\lceil k /\lfloor(m+1) / 3\rfloor\rceil-1))}{2}\right\rfloor\right) \\
& -3-3\left\lfloor\frac{m+1}{3}\right\rfloor\left\lfloor\frac{i-1}{2}\right\rfloor \\
& =3 k+m-3 k+3+3\left\lfloor\frac{m+1}{3}\right\rfloor\left(\left\lfloor\frac{k}{\lfloor(m+1) / 3\rfloor}\right\rfloor-1\right)-3-3\left\lfloor\frac{m+1}{3}\right\rfloor\left\lfloor\frac{i-1}{2}\right\rfloor \\
& =m+3\left\lfloor\frac{m+1}{3}\right\rfloor\left(\left\lceil\frac{k}{\lfloor(m+1) / 3\rfloor}\right]-1-\left\lfloor\frac{i-1}{2}\right\rfloor\right) \\
& =m+3\left\lfloor\frac{m+1}{3}\right\rfloor\left(\left\lceil\frac{k}{\lfloor(m+1) / 3\rfloor}\right\rfloor-1-\left\lfloor\frac{i-1}{2}\right\rfloor\right) \\
& =m \text {, } \\
& \text { For } i=2\left\lceil\frac{k}{\lfloor(m+1) / 3\rfloor}\right\rceil+1 ; j=1,2, \ldots, m+1, \\
& \mathrm{Wt}_{(1,1,0)}\left(f_{i+1}^{j}\right)-\mathrm{Wt}_{(1,1,0)}\left(f_{i}^{j}\right)=\phi\left(v_{i+1}^{j}\right)+\phi\left(v_{i+1}^{j+1}\right)+\phi\left(v_{i+2}^{j}\right)+\phi\left(v_{i+2}^{j+1}\right)+\phi\left(v_{i+1}^{j} v_{i+1}^{j+1}\right)+\phi\left(v_{i+1}^{j} v_{i+2}^{j}\right) \\
& +\phi\left(v_{i+2}^{j} v_{i+2}^{j+1}\right)+\phi\left(v_{i+1}^{j+1} v_{i+2}^{j+1}\right)-\phi\left(v_{i}^{j}\right)-\phi\left(v_{i}^{j+1}\right)-\phi\left(v_{i+1}^{j}\right)-\phi\left(v_{i+1}^{j+1}\right)-\phi\left(v_{i}^{j} v_{i}^{j+1}\right)-\phi\left(v_{i}^{j} v_{i+1}^{j}\right) \\
& -\phi\left(v_{i+1}^{j} v_{i+1}^{j+1}\right)-\phi\left(v_{i}^{j+1} v_{i+1}^{j+1}\right) \text {, } \\
& \mathrm{Wt}_{(1,1,0)}\left(f_{i+1}^{j}\right)-\mathrm{Wt}_{(1,1,0)}\left(f_{i}^{j}\right)=k+k+k+\left\lceil\frac{j}{2}\right\rceil+\left\lceil\frac{j+1}{2}\right\rceil+2\left(m-3 k+3+3\left\lfloor\frac{m+1}{3}\right\rfloor\left(\left\lceil\frac{k}{\lfloor(m+1) / 3\rfloor}\right\rceil-1\right)\right)
\end{aligned}
$$




$$
\begin{aligned}
& +\left\lceil\frac{1}{2}\left(i+1-2\left\lceil\frac{k}{\lfloor(m+1) / 3\rfloor}\right\rceil-1\right)\right\rceil\left(\left\lfloor\frac{m}{2}\right\rfloor+\left\lceil\frac{m}{2}\right\rceil\right) \\
& +\left\lfloor\frac{1}{2}\left(i+1-2\left\lceil\frac{k}{\lfloor(m+1) / 3\rfloor}\right\rceil-1\right)\right\rfloor\left(\left\lceil\frac{m}{2}\right\rceil+\left\lfloor\frac{m}{2}\right\rfloor\right) \\
& -k-k-k-\left\lceil\frac{j}{2}\right\rceil-\left\lceil\frac{j+1}{2}\right\rceil-\left\lceil\frac{1}{2}\left(i-2\left\lceil\frac{k}{\lfloor(m+1) / 3\rfloor}\right\rceil+1\right)\right\rceil \\
& \cdot\left(\left\lfloor\frac{(m-3 k+3+3\lfloor(m+1) / 3\rfloor(\lceil k /\lfloor(m+1) / 3\rfloor\rceil-1))}{2}\right\rfloor\right. \\
& \left.+\left\lceil\frac{(m-3 k+3+3\lfloor(m+1) / 3\rfloor(\lceil k /\lfloor(m+1) / 3\rfloor\rceil)-1)}{2}\right\rceil\right) \\
& -\left\lfloor\frac{1}{2}\left(i-2\left\lceil\frac{k}{\lfloor(m+1) / 3\rfloor}\right\rceil+1\right)\right\rfloor \\
& \cdot\left(\left\lceil\frac{(m-3 k+3+3\lfloor(m+1) / 3\rfloor(\lceil k /\lfloor(m+1) / 3\rfloor\rceil-1))}{2}\right\rceil\right. \\
& \left.+\left\lfloor\frac{(m-3 k+3+3\lfloor(m+1) / 3\rfloor(\lceil k /\lfloor(m+1) / 3\rfloor\rceil)-1)}{2}\right\rfloor\right) \\
& =2\left(m-3 k+3+3\left\lfloor\frac{m+1}{3}\right\rfloor\left(\left\lceil\frac{k}{\lfloor(m+1) / 3\rfloor}\right\rceil-1\right)\right) \\
& +(1)(m)-(1)\left(m-3 k+3+3\left[\frac{m+1}{3}\right]\left(\left[\frac{k}{\lfloor(m+1) / 3\rfloor}\right]-1\right)\right) \\
& -(1)\left(m-3 k+3+3\left\lfloor\frac{m+1}{3}\right\rfloor\left(\left[\frac{k}{\lfloor(m+1) / 3\rfloor}\right\rceil-1\right)\right) \\
& =m \text {, } \\
& \text { For } i=2\left\lceil\frac{k}{\lfloor(m+1) / 3\rfloor}\right\rceil+2, \ldots, n ; j=1,2, \ldots, m+1, \\
& \mathrm{Wt}_{(1,1,0)}\left(f_{i+1}^{j}\right)-\mathrm{Wt}_{(1,1,0)}\left(f_{i}^{j}\right)=\phi\left(v_{i+1}^{j}\right)+\phi\left(v_{i+1}^{j+1}\right)+\phi\left(v_{i+2}^{j}\right)+\phi\left(v_{i+2}^{j+1}\right)+\phi\left(v_{i+1}^{j} v_{i+1}^{j+1}\right)+\phi\left(v_{i+1}^{j} v_{i+2}^{j}\right) \\
& +\phi\left(v_{i+2}^{j} v_{i+2}^{j+1}\right)+\phi\left(v_{i+1}^{j+1} v_{i+2}^{j+1}\right)-\phi\left(v_{i}^{j}\right)-\phi\left(v_{i}^{j+1}\right) \\
& -\phi\left(v_{i+1}^{j}\right)-\phi\left(v_{i+1}^{j+1}\right)-\phi\left(v_{i}^{j} v_{i}^{j+1}\right)-\phi\left(v_{i}^{j} v_{i+1}^{j}\right) \\
& -\phi\left(v_{i+1}^{j} v_{i+1}^{j+1}\right)-\phi\left(v_{i}^{j+1} v_{i+1}^{j+1}\right) \text {, } \\
& \mathrm{Wt}_{(1,1,0)}\left(f_{i+1}^{j}\right)-\mathrm{Wt}_{(1,1,0)}\left(f_{i}^{j}\right)=k+k+k+\left\lceil\frac{j}{2}\right\rceil+\left\lceil\frac{j+1}{2}\right\rceil+2\left(m-3 k+3+3\left\lfloor\frac{m+1}{3}\right\rfloor\left(\left\lceil\frac{k}{\lfloor(m+1) / 3\rfloor}\right\rceil-1\right)\right) \\
& +\left\lceil\frac{1}{2}\left(i+1-2\left\lceil\frac{k}{\lfloor(m+1) / 3\rfloor}\right\rceil-1\right)\right\rceil\left(\left\lfloor\frac{m}{2}\right\rfloor+\left\lceil\frac{m}{2}\right\rceil\right) \\
& +\left\lfloor\frac{1}{2}\left(i+1-2\left\lceil\frac{k}{\lfloor(m+1) / 3\rfloor}\right\rceil-1\right)\right\rfloor\left(\left\lceil\frac{m}{2}\right\rceil+\left\lfloor\frac{m}{2}\right\rfloor\right)-k-k
\end{aligned}
$$




$$
\begin{aligned}
& -k-\left\lceil\frac{j}{2}\right\rceil-\left\lceil\frac{j+1}{2}\right\rceil-2\left(m-3 k+3+3\left\lfloor\frac{m+1}{3}\right\rfloor\left(\left\lceil\frac{k}{\lfloor(m+1) / 3\rfloor}\right\rceil-1\right)\right) \\
& -\left\lceil\frac{1}{2}\left(i-2\left\lceil\frac{k}{\lfloor(m+1) / 3\rfloor}\right\rceil-1\right)\right\rceil\left(\left\lfloor\frac{m}{2}\right\rfloor+\left\lceil\frac{m}{2}\right\rceil\right)-\left\lfloor\frac{1}{2}\left(i-2\left\lceil\frac{k}{\lfloor(m+1) / 3\rfloor}\right\rceil-1\right)\right\rfloor\left(\left\lceil\frac{m}{2}\right\rceil+\left\lfloor\frac{m}{2}\right\rfloor\right) \\
& =m\left\lceil\frac{1}{2}\left(i+1-2\left\lceil\frac{k}{\lfloor(m+1) / 3\rfloor}\right\rceil-1\right)\right\rceil \\
& +m\left\lfloor\frac{1}{2}\left(i+1-2\left\lceil\frac{k}{\lfloor(m+1) / 3\rfloor}\right\rceil-1\right)\right\rfloor-m\left\lceil\frac{1}{2}\left(i-2\left\lceil\frac{k}{\lfloor(m+1) / 3\rfloor}\right\rceil-1\right)\right\rceil \\
& -m\left\lfloor\frac{1}{2}\left(i-2\left\lceil\frac{k}{\lfloor(m+1) / 3\rfloor}\right\rceil-1\right)\right\rfloor \\
& =m\left(\left\lceil\frac{(i+1-2\lceil k /\lfloor(m+1) / 3\rfloor\rceil-1)}{2}\right\rceil+\left\lfloor\frac{(i+1-2\lceil k /\lfloor(m+1) / 3\rfloor\rceil-1)}{2}\right\rfloor\right) \\
& -m\left(\left\lceil\frac{(i-2\lceil k /\lfloor(m+1) / 3\rfloor\rceil-1)}{2}\right\rceil+\left\lfloor\frac{(i-2\lceil k /\lfloor(m+1) / 3\rfloor\rceil-1)}{2}\right\rfloor\right) \\
& =m\left(i+1-2\left\lceil\frac{k}{\lfloor(m+1) / 3\rfloor}\right\rceil-1\right)-m\left(i-2\left\lceil\frac{k}{\lfloor(m+1) / 3\rfloor}\right\rceil-1\right) \\
& =m\left(i+1-2\left\lceil\frac{k}{\lfloor(m+1) / 3\rfloor}\right\rceil-1-i+2\left\lceil\frac{k}{\lfloor(m+1) / 3\rfloor}\right\rceil+1\right) \\
& =m \text {. }
\end{aligned}
$$

Example 1. The total face irregularity strength of grid graph $G_{6}^{3}$, under a $k$-labeling of type $(1,1,0)$ is 4 .

Proof. The graph under consideration is $G_{6}^{3}=P_{7} \square P_{4}$. Figure 2 is a 4 -labeling of type $(1,1,0)$ for the grid graph $G_{6}^{3}$, and it will help us in calculating total face irregularity strength in different intervals of the grid graph.
Here, $k=\lceil(18+7) / 8\rceil=4,\lfloor(m+1) / 3\rfloor=1,\lceil k /\lfloor(m+$ $1) / 3\rfloor\rceil=4$, and $m-2\lfloor(m+1) / 3\rfloor=1$

In order to show that $\operatorname{tfs}_{(1,1,0)}\left(G_{6}^{3}\right)=4$, it is sufficient to prove that all the horizontal differences in face weights are 1 and all the vertical differences in face weights are 3 . Now, we prove these results.

Horizontal differences in face weights can be calculated as follows:

For $i=1,2,3,4,5,6$ and $j=1,2,3,4$,

$$
\begin{aligned}
\mathrm{Wt}_{(1,1,0)}\left(f_{i}^{j+1}\right)-\mathrm{Wt}_{(1,1,0)}\left(f_{i}^{j}\right)= & \phi\left(v_{i}^{j+1}\right)+\phi\left(v_{i}^{j+2}\right)+\phi\left(v_{i+1}^{j+1}\right)+\phi\left(v_{i+1}^{j+2}\right)+\phi\left(v_{i}^{j+1} v_{i}^{j+2}\right)+\phi\left(v_{i}^{j+1} v_{i+1}^{j+1}\right) \\
& +\phi\left(v_{i+1}^{j+1} v_{i+1}^{j+2}\right)+\phi\left(v_{i}^{j+2} v_{i+1}^{j+2}\right)-\phi\left(v_{i}^{j}\right)-\phi\left(v_{i}^{j+1}\right)-\phi\left(v_{i+1}^{j}\right)-\phi\left(v_{i+1}^{j+1}\right)-\phi\left(v_{i}^{j} v_{i}^{j+1}\right)-\phi\left(v_{i}^{j} v_{i+1}^{j}\right) \\
& -\phi\left(v_{i+1}^{j} v_{i+1}^{j+1}\right)-\phi\left(v_{i}^{j+1} v_{i+1}^{j+1}\right) \\
= & \left\lceil\frac{j+2}{2}\right\rceil-\left\lceil\frac{j}{2}\right\rceil \\
= & 1, \quad \text { for every value of } j,
\end{aligned}
$$




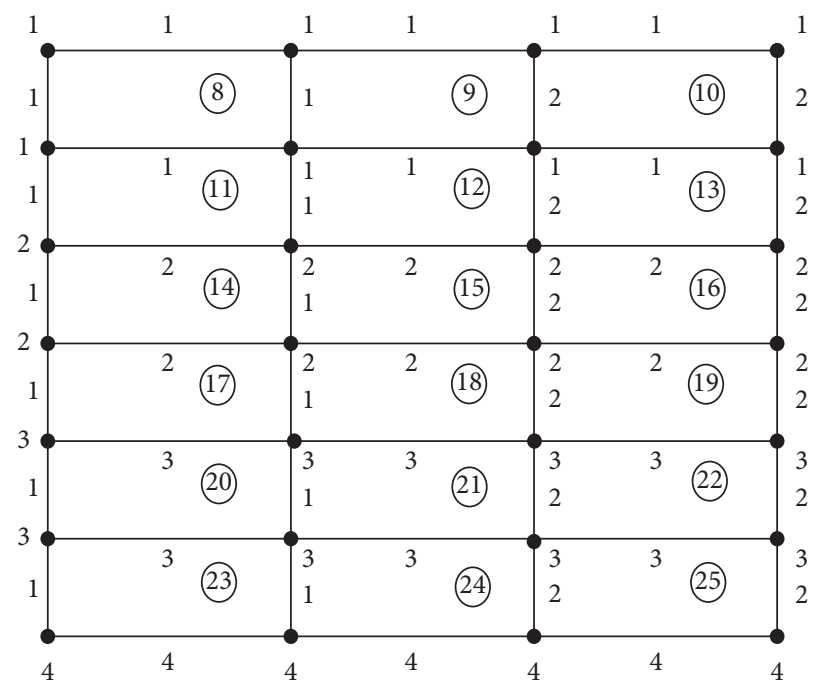

Figure 2: Total face irregular 4-labeling of the $(1,1,0)$ of grid graph $G_{6}^{3}$.

For $i=7$ and $j=1,2, \ldots, m+1$,

$$
\begin{aligned}
\mathrm{Wt}_{(1,1,0)}\left(f_{i}^{j+1}\right)-\mathrm{Wt}_{(1,1,0)}\left(f_{i}^{j}\right)= & \phi\left(v_{i}^{j+1}\right)+\phi\left(v_{i}^{j+2}\right)+\phi\left(v_{i+1}^{j+1}\right)+\phi\left(v_{i+1}^{j+2}\right)+\phi\left(v_{i}^{j+1} v_{i}^{j+2}\right)+\phi\left(v_{i}^{j+1} v_{i+1}^{j+1}\right) \\
& +\phi\left(v_{i+1}^{j+1} v_{i+1}^{j+2}\right)+\phi\left(v_{i}^{j+2} v_{i+1}^{j+2}\right)-\phi\left(v_{i}^{j}\right)-\phi\left(v_{i}^{j+1}\right)-\phi\left(v_{i+1}^{j}\right)-\phi\left(v_{i+1}^{j+1}\right)-\phi\left(v_{i}^{j} v_{i}^{j+1}\right)-\phi\left(v_{i}^{j} v_{i+1}^{j}\right) \\
& -\phi\left(v_{i+1}^{j} v_{i+1}^{j+1}\right)-\phi\left(v_{i}^{j+1} v_{i+1}^{j+1}\right) \\
= & \left\lceil\frac{j+2}{2}\right\rceil-\left\lceil\frac{j}{2}\right\rceil
\end{aligned}
$$

$=1$, for all values of $j$.

Vertical differences in face weights can be calculated as follows:

For $i=1,2,3,4,5,6$ and $j=1,2,3,4$,

$$
\begin{aligned}
\mathrm{Wt}_{(1,1,0)}\left(f_{i+1}^{j}\right)-\mathrm{Wt}_{(1,1,0)}\left(f_{i}^{j}\right)= & \phi\left(v_{i+1}^{j}\right)+\phi\left(v_{i+1}^{j+1}\right)+\phi\left(v_{i+2}^{j}\right)+\phi\left(v_{i+2}^{j+1}\right)+\phi\left(v_{i+1}^{j} v_{i+1}^{j+1}\right)+\phi\left(v_{i+1}^{j} v_{i+2}^{j}\right) \\
& +\phi\left(v_{i+2}^{j} v_{i+2}^{j+1}\right)+\phi\left(v_{i+1}^{j+1} v_{i+2}^{j+1}\right)-\phi\left(v_{i}^{j}\right)-\phi\left(v_{i}^{j+1}\right)-\phi\left(v_{i+1}^{j}\right)-\phi\left(v_{i+1}^{j+1}\right)-\phi\left(v_{i}^{j} v_{i}^{j+1}\right)-\phi\left(v_{i}^{j} v_{i+1}^{j}\right) \\
& -\phi\left(v_{i+1}^{j} v_{i+1}^{j+1}\right)-\phi\left(v_{i}^{j+1} v_{i+1}^{j+1}\right) \\
= & 3\left\lfloor\frac{m+1}{3}\right\rfloor \\
= & 3,
\end{aligned}
$$

For $i=7 ; j=1,2,3,4$, 


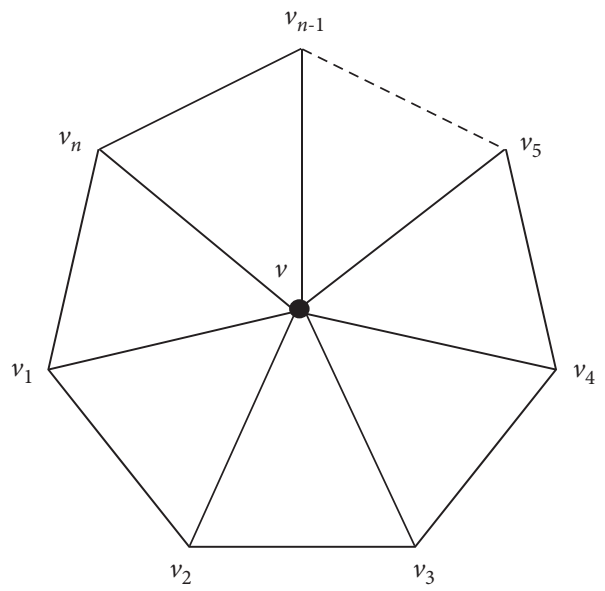

FIgURE 3: Wheel graph $W_{n}$.

$$
\begin{aligned}
\mathrm{Wt}_{(1,1,0)}\left(f_{i+1}^{j}\right)-\mathrm{Wt}_{(1,1,0)}\left(f_{i}^{j}\right)= & \phi\left(v_{i+1}^{j}\right)+\phi\left(v_{i+1}^{j+1}\right)+\phi\left(v_{i+2}^{j}\right)+\phi\left(v_{i+2}^{j+1}\right)+\phi\left(v_{i+1}^{j} v_{i+1}^{j+1}\right)+\phi\left(v_{i+1}^{j} v_{i+2}^{j}\right) \\
& +\phi\left(v_{i+2}^{j} v_{i+2}^{j+1}\right)+\phi\left(v_{i+1}^{j+1} v_{i+2}^{j+1}\right)-\phi\left(v_{i}^{j}\right)-\phi\left(v_{i}^{j+1}\right)-\phi\left(v_{i+1}^{j}\right)-\phi\left(v_{i+1}^{j+1}\right)-\phi\left(v_{i}^{j} v_{i}^{j+1}\right)-\phi\left(v_{i}^{j} v_{i+1}^{j}\right) \\
& -\phi\left(v_{i+1}^{j} v_{i+1}^{j+1}\right)-\phi\left(v_{i}^{j+1} v_{i+1}^{j+1}\right) \\
= & m+3\left\lfloor\frac{m+1}{3}\right\rfloor\left(\left[\frac{k}{\lfloor(m+1) / 3\rfloor}\right]-1-\left\lfloor\left[\frac{k}{\lfloor(m+1) / 3\rfloor}\right\rfloor-1\right\rfloor\right) \\
= & 3 .
\end{aligned}
$$

It shows that all the differences of horizontal faces are equal to one and all the differences of vertical faces are equal to $m$. Hence, total face irregularity strength of grid graph $G_{6}^{3}$ is 4 .

Theorem 4. . Let $W_{n}$ be a wheel graph with $n+1$ vertices, where $n \geq 3$. Then, under a total $k$-labeling of type $(1,1,0)$, we have

$$
\operatorname{tfs}\left(W_{n}\right)=\left\lceil\frac{n+4}{5}\right\rceil
$$

Proof. Let $W_{n}$ be a wheel graph with $n+1$ vertices, then by the definition of wheel graph, the total number of edges will be $2 n$ and the total number of faces will be $n+1$, that is,

$$
\begin{aligned}
& \left|E\left(W_{n}\right)\right|=2 n, \\
& \left|F\left(W_{n}\right)\right|=n+1 .
\end{aligned}
$$

As we see that a wheel graph has 3-sided internal faces and external face, so by using Theorem 2, we have

$$
\operatorname{tfs}\left(W_{n}\right) \geq\left\lceil\frac{n+4}{5}\right\rceil \text {. }
$$

In Figure 3, $v$ is the vertex in the center of wheel graph $W_{n}$ which is connecting to all the vertices $v_{i}$ for $1 \leq i \leq n$. Similarly, for $1 \leq i \leq n-1$, the edges of the wheel graph can be constructed as $E\left(W_{n}\right)=\left\{v v_{i}, v_{i} v_{i+1}, v v_{n}, v_{1} v_{n}\right\}$. Also for $1 \leq i \leq n-1$, there will be exterior face, the $n$th interior face can be written as $f\left(W_{n}\right)=\left\{v v_{1} v_{n} v\right\}$, and other all 3-sided interior faces can be written as $f\left(W_{n}\right)=\left\{v v_{i} v_{i+1} v\right\}$. Let us define a total $k$-labeling $\phi: V \cup E \longrightarrow\{1,2,3, \ldots,\lceil(n+$ 4)/57\}.

In Figure 4, we consider a finite wheel graph $W_{3}$ which is labeled under a 2 -labeling of type $(1,1,0)$. So, for $1 \leq i \leq 3$, we have

$$
\begin{gathered}
\phi(v)=\phi\left(v_{2}\right)=\phi\left(v v_{i}\right)=\phi\left(v_{1} v_{2}\right)=1, \\
\phi\left(v_{1}\right)=\phi\left(v_{3}\right)=\phi\left(v_{1} v_{3}\right)=\phi\left(v_{2} v_{3}\right)=2 .
\end{gathered}
$$

Weight of exterior face will be

$$
\mathrm{Wt}\left(f_{\text {exterior }}\right)=10 \text {. }
$$

Weight of interior faces will be

$$
\mathrm{Wt}\left(f_{i}\right)=i+6
$$

Now, let us talk about the graphs except $W_{3}$ for which we define the labeling as $\phi(v)=1$ :

(i) For $1 \leq i \leq\lceil n / 2\rceil+1$, we have $\phi\left(v_{i}\right)=\lceil 2 i / 5\rceil$

(ii) For $\lceil n / 2\rceil+2 \leq i \leq n$, we have $\phi\left(v_{i}\right)=\lceil 2(n-i+$ 1) $/ 5\rceil+1$

(iii) For $1 \leq i \leq\lceil n / 2\rceil+1$, we have $\phi\left(v v_{i}\right)=\lceil(2 i-1) / 5\rceil$ 


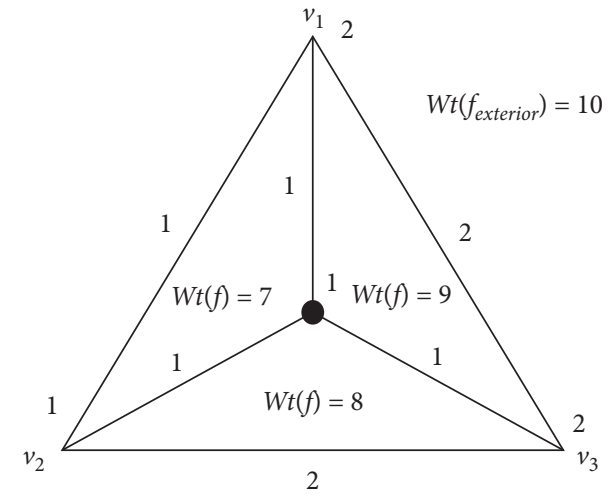

FIGURE 4: 2-labeling of a wheel graph $W_{3}$.

(iv) For $\lceil n / 2\rceil+2 \leq i \leq n$, we have $\phi\left(v v_{i}\right)=\lceil(2(n-$ i) +1$) / 5\rceil+1$

(v) For $i=1$, we have $\phi\left(v_{1} v_{n}\right)=1$

(vi) For $2 \leq i \leq\lceil n / 2\rceil$, we have $\phi\left(v_{i} v_{i+1}\right)=\lceil(2 i-2) / 5\rceil$

(vii) For $i=\lceil n / 2\rceil+1$, where $n \equiv m(\bmod 6)$ in which $m=\{2,3,4,5\}$, we have $\phi\left(v_{i} v_{i+1}\right)=\lceil(2 i-2) / 5\rceil$

(viii) For $i=\lceil n / 2\rceil+1$ where $n \equiv m(\bmod 6)$ in which $m \in\{0,1\}$, we have $\phi\left(v_{i} v_{i+1}\right)=\lceil(n+4) / 5\rceil$

(xi) For $i=\lceil n / 2\rceil+2 \leq i \leq n-1$, we have $\phi\left(v_{i} v_{i+1}\right)=\lceil(2$ $(n-i)-2) / 5\rceil+1$

For weights of the wheel graph $W_{n}$, we proceed as follows:

(i) For $i=1$, we have $\mathrm{Wt}\left(f_{i}\right)=6$

(ii) For $2 \leq i \leq\lceil(n+1) / 2\rceil$, we have $\mathrm{Wt}\left(f_{i}\right)=2 i+3$

(iii) For $\lceil(n+1) / 2\rceil+1 \leq i \leq n$, we have $\operatorname{Wt}\left(f_{i}\right)=2(n-$ i) +8

(iv) For external weight, we will use $\mathrm{Wt}\left(f_{\text {exterior }}\right) \geq$ $2 n+8$

We can easily observe that under the $k$-labeling $\phi$ of type $(1,1,0)$, the minimum $k$ for which the wheel graph admits total face irregular strength is $\lceil(n+4) / 5\rceil$. Hence,

$$
\operatorname{tfs}\left(W_{n}\right)=\left\lceil\frac{n+4}{5}\right\rceil \text {. }
$$

\section{Conclusion}

We investigated total face irregularity strength of generalized plane grid graphs $G_{n}^{m}$ and wheel graphs $W_{n}$ under a graph $k$-labeling of type $(\alpha, \beta, \gamma)$ where $\alpha, \beta \in\{0,1\}$. This work was based on the bright idea of finding face irregularity strength of ladder graphs by Martin Baca et al. [14]. In this article, we worked on the total face irregularity strength of grid and wheel graphs. We labeled graph vertices and graph edges but focussed on estimating face weights of graphs to prove the sharpness of $k$-labeling. We derived generalized formulas by considering graphs with different values of $n, m$, $\lfloor(m+1) / 3\rfloor$, and $m-2\lfloor(m+1) / 3\rfloor$. Also, we verified the final results with example. In future, total and entire face irregular strength of some more products of different plane graphs can be investigated under $k$-labeling of type $(\alpha, \beta, \gamma)$.

\section{Data Availability}

No data were used to support this study.

\section{Conflicts of Interest}

The authors declare that they have no conflicts of interest.

\section{References}

[1] G. Chartrand, M. S. Jacobson, J. Lehel, O. R. Oellermann, S. Ruiz, and F. Saba, "Irregular networks," Congressus Numerantium, vol. 64, pp. 187-192, 1988.

[2] T. Nierhoff, "A tight bound on the irregularity strength of graphs," SIAM Journal on Discrete Mathematics, vol. 13, no. 3, pp. 313-323, 2000.

[3] M. Kalkowski, M. Karoński, and F. Pfender, "A new upper bound for the irregularity strength of graphs," SIAM Journal on Discrete Mathematics, vol. 25, no. 3, pp. 1319-1321, 2011.

[4] A. Ahmad, M. Bača, and M. K. Siddiqui, "On edge irregular total labeling of categorical product of two cycles," Theory of Computing Systems, vol. 54, no. 1, pp. 1-12, 2014.

[5] D. Amar and O. Togni, "Irregularity strength of trees," Discrete Mathematics, vol. 190, no. 1-3, pp. 15-38, 1998.

[6] J. H. Dimitz, D. K. Garnick, and A. Gyárfás, "On the irregularity strength of the $m \times n$ grid," Journal of Graph Theory, vol. 16, pp. 355-374, 1992.

[7] A. Gyárfás, "The irregularity strength of $K_{m, m}$ is 4 for odd," Discrete Mathematics, vol. 71, pp. 273-274, 1998.

[8] A. Frieze, R. J. Gould, M. Karoski, and F. Pfender, “On graph irregularity strength," Journal of Graph Theory, vol. 41, no. 2, pp. 120-137, 2002.

[9] S. Jendrol', J. Miškuf, and R. Soták, “Total edge irregularity strength of complete graphs and complete bipartite graphs," Discrete Mathematics, vol. 310, pp. 400-407, 2010.

[10] Nurdin, E. T. Baskoro, A. N. M. Salman, and N. N. Gaos, "On the total vertex irregularity strength of trees," Discrete Mathematics, vol. 310, no. 21, pp. 3043-3048, 2010.

[11] M. Bača and M. K. Siddiqui, "Total edge irregularity strength of generalized prism," Applied Mathematics and Computation, vol. 235, pp. 168-173, 2014.

[12] A. Ahmad, O. B. S. Al-Mushayt, and M. Bača, "On edge irregularity strength of graphs," Applied Mathematics and Computation, vol. 243, pp. 607-610, 2014.

[13] G. Ebert, J. Hemmeter, F. Lazebnik, and A. Woldar, "Irregularity strengths of certain graphs," Congressus Numerantium, vol. 71, pp. 39-52, 1990.

[14] M. Baca, S. Jendrol', M. Miller, and J. Ryan, "On irregular total labellings,” Discrete Mathematics, vol. 307, pp. 1378-1388, 2007.

[15] M. Bača, A. Ovais, A. Semaničová-Feňovčiková, and I. N. Suparta, "On face irregular evaluations of plane graphs," Discussiones Mathematicae Graph Theory, vol. 22, 2020.

[16] M. Bača, S. Jendroī, K. Kathiresan, and K. Muthugurupackiam, "Entire labeling of plane graphs," Applied Mathematics \& Information Sciences, vol. 9, no. 1, pp. 263-267, 2015. 